\title{
Has the Twin Paradox Really Been Resolved?
}

\author{
Pirooz Mohazzabi, Qinghua Luo \\ Department of Mathematics and Physics, University of Wisconsin-Parkside, Kenosha, WI, USA \\ Email: mohazzab@uwp.edu
}

How to cite this paper: Mohazzabi, P. and Luo, Q.H. (2021) Has the Twin Paradox Really Been Resolved? Journal of Applied Mathematics and Physics, 9, 2187-2192. https://doi.org/10.4236/jamp.2021.99138

Received: July 29, 2021

Accepted: August 31, 2021

Published: September 3, 2021

Copyright (c) 2021 by author(s) and Scientific Research Publishing Inc. This work is licensed under the Creative Commons Attribution International License (CC BY 4.0).

http://creativecommons.org/licenses/by/4.0/

\begin{abstract}
Despite several attempts that have been made to explain the twin paradox, which are based on asymmetry and acceleration, it is shown that none of these explanations can resolve the paradox in the general case. Three specific examples of the twin paradox are provided in which the system is completely symmetric; consequently the existing explanations are not able to resolve the paradox.
\end{abstract}

\section{Keywords}

Twin Paradox, Asymmetry, Acceleration, Relativity, Lorentz Transformation

\section{Introduction}

Perhaps the most perplexing and controversial thought experiment in physics, and particularly in the theory of special relativity, is the twin paradox, which was realized by Einstein himself in 1905. The description of this paradox can be found in many places in the literature [1]-[8].

The paradox is based on the concept of time dilation in the theory of special relativity. If observer $O^{\prime}$ moves with velocity $v$ relative to observer $O$, then the time interval between two events taking place at the same point in the frame of reference of the moving observer $\Delta t^{\prime}$ (known as the proper time) is related to the same time $\Delta t$ measured by observer $O$ according to [1] [2] [3] [9]

$$
\Delta t=\frac{\Delta t^{\prime}}{\sqrt{1-v^{2} / c^{2}}}
$$

where $c$ is the speed of light. This result is a direct consequence of Einstein's second postulate of special relativity, which states that the speed of light is the same in all inertial frames of reference [10].

Because $\sqrt{1-v^{2} / c^{2}}<1$, the time measured in the rest frame will be greater than that measured in the moving frame, $\Delta t>\Delta t^{\prime}$. Furthermore, these times 
correspond to any process, including the working of a clock and the biological evolution of the human body. Consequently, the clock in the moving frame falls behind and ticks slower than that in the rest frame and the traveler will age less than the person in the rest frame.

Equation (1) and its consequences described above are known as kinematic time dilation and are the results of special relativity. In addition, according to the theory of general relativity, time also dilates due to gravitational field. Thus, clocks tick faster in high gravitational field compared to low gravitational field. This is known as gravitational time dilation [11]. These results have been demonstrated by experiments and their validity has been established. Perhaps the most compelling evidence of the correctness of time dilation is the well-known experiment of Hafele and Keating [12]. In their experiment, they flew four cesium beam clocks around the world eastward and westward, and measured the time lost and gained by them compared to a clock on Earth. They found results that were in good agreement with those predicted theoretically.

The twin paradox is the consequence of the following thought experiment. System $O$ is at rest and system $O^{\prime}$ is moving. Therefore, the clock in $O^{\prime}$ ticks slower than that in $O$. Thus, for example, if the two clocks are initially synchronized to read $t=t^{\prime}=0$, after a while they may show $t=10$ (some arbitrary unit of time) but $t^{\prime}=6$. Therefore, an observer moving with system $O^{\prime}$ will be younger than that in system $O$. However, as seen by the observer in $O^{\prime}$, she is at rest and system $O$ is moving away from her. Therefore, according the observer in $O^{\prime}$, the observer in $O$ should be younger. This is the foundation of the twin paradox, which is stated as follows: Twin $A$ is on Earth and twin $B$ travels to a distant star with a speed close to the speed of light. Afterward, she returns to Earth with the same speed. When they reunite, according to twin $A$, twin $B$ must be younger, but according to twin $B$, twin $A$ must be younger.

\section{Attempts to Explain the Twin Paradox}

Many attempts have been made to explain the twin paradox, which fall in two categories; one based on asymmetry and the other on acceleration. Each argument, however, is based on the following description of the paradox. Twins $A$ and $B$ synchronize their clocks on Earth, $t_{A}=t_{B}=0$. Then twin $B$ departs toward a distance star $S$ with a speed $v$ relative to Earth, while twin $A$ remains at rest on Earth, as shown in Figure 1. Upon arriving at star $S$, twin $B$ turns around and returns to Earth with the same speed. When the twins reunite, according to Equation (1), twin $B$ who was moving relative to $A$ must have aged less. But from the point of view of $B$, twin $A$ was moving during the process, and she

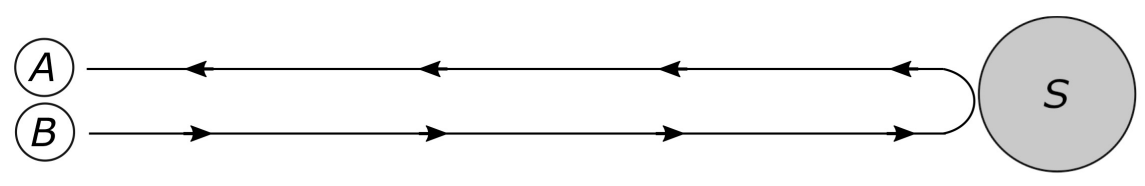

Figure 1. A diagram for the commonly described twin paradox. 
must be the younger one. This is because each twin sees the other twin as moving. This scenario is generally referred to as the twin paradox.

To resolve the twin paradox, some authors resort to asymmetry argument. They argue that twin $B$ does not remain in a single inertial frame of reference during the entire process; traveling toward the star she is in one frame of reference while coming back she is in another one [1] [13] [14] [15] [16]. In a second school of thought, some authors argue that the twin who leaves the Earth undergoes acceleration whereas the one who stays on Earth is not accelerating [4] [17] [18]. Some even argue that time dilation equation is not valid if the reference system accelerates [3].

\section{Why the Current Explanations Are Unsatisfactory}

Looking at the specific example of twin paradox described in Figure 1, where one twin is stationary and the other accelerates towards a star and back, one can of course resort to asymmetry arguments. Thus, one can argue that one twin stays in a single reference frame whereas the other one does not. Or that one twin accelerates whereas the other one does not. However, these arguments apply only to asymmetric situations as that described here. There are many other examples of the twin paradox, where the system is completely symmetric in every respect, consequently the paradox cannot be explained by such simple-minded arguments. Here are a few of them.

\subsection{Twins Approaching Each Other}

Consider twins, $A$ and $B$, both initially at rest with respect to an inertial frame and separated by distance $d$. They synchronize their clocks according to the following method. When the clock of twin $A$ reads $t_{A}=0$, she sends a light signal towards twin $B$. This light signal takes a time $d / c$ to reach twin $B$. So, when twin $B$ receives the light signal, she sets her clock to $t_{B}=d / c$ [2]. Then at a time that the two twins had previously agreed upon, they start moving towards each other with equal accelerations relative to an inertial frame $O$ at their midpoint. The accelerations are very large but take place in a very short time (essentially a Dirac $\delta$ function) resulting in relativistic speeds. The two twins then start moving towards each other, each with a constant speed $v$ relative to the other, as shown in Figure 2.

According to twin $A$, twin $B$ is moving with speed $v$. Therefore when they reach each other at the midpoint $O$, the clock of $B$ should show a shorter time than the clock of $A$, i.e., $t_{B}<t_{A}$. On the other hand, according to twin $B$, twin $A$ is moving with speed $v$. Therefore when they reach each other, the clock of $A$ should show a shorter time than the clock of $B$, i.e., $t_{A}<t_{B}$. In this situation, the

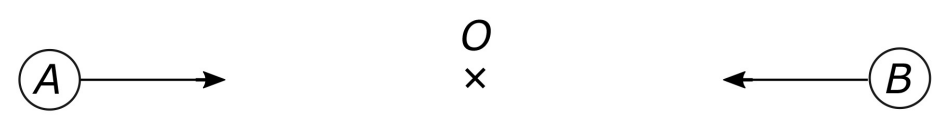

Figure 2. Twins $A$ and $B$ approaching each other with relative speed $v$. 
system is completely symmetric; neither twin leaves her reference frame, and both have the same initial acceleration. Therefore, none of the suggested explanations can resolve the paradox in this case.

\subsection{An Object Splitting into Two Parts}

Consider two equal-mass objects $A$ and $B$, held together with some explosive charge between them, as shown in Figure 3. There is a clock on each object that is synchronized. The charge explodes, sending the objects in the opposite directions, each moving with a relativistic speed $v$ relative to the other. The objects make elastic collisions with the solid walls shown and return. Since the center of mass remains the same as the initial position of the objects, they will meet again at that point. During the initial explosion of the charge and during the elastic collisions with the walls, the two objects undergo nearly instantaneous accelerations, which constitutes a negligible amount of time compared to the entire process.

Now, relative to object $A$, object $B$ was moving. Therefore, when they meet $t_{B}<t_{A}$. On the other hand, relative to $B$, object $A$ was moving, therefore, $t_{A}<t_{B}$. Again, the twin paradox stands and none of the suggested explanations can resolve it as the process is completely symmetric.

\subsection{Binary Star Systems}

Consider two equal-mass stars in a binary star system, rotating about their common center of mass, as shown in Figure 4. A light flashes at their center of mass and when it reaches the stars, they synchronize their clacks to $t_{A}=t_{B}=0$. At a later time the light flashes again and the two stars record the reading of their clocks. Let us see what happens when the two readings are compared later.

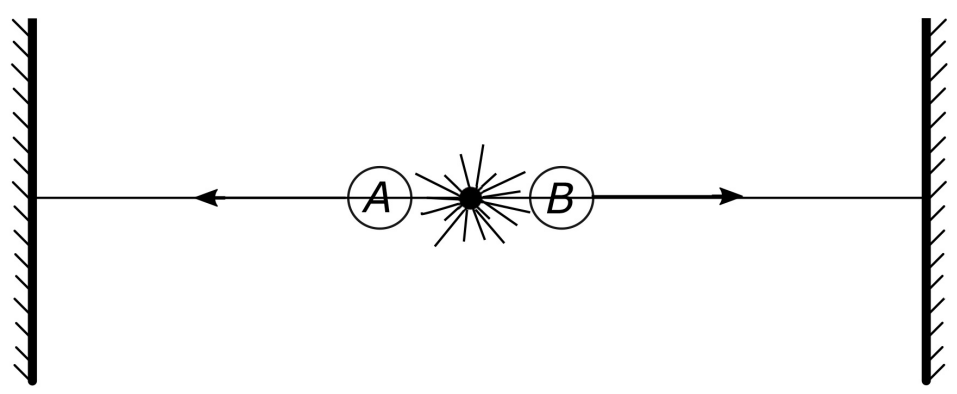

Figure 3. Two objects held together with a charge between them. When the charge explodes, the objects move away from each other and bounce back elastically from the walls.

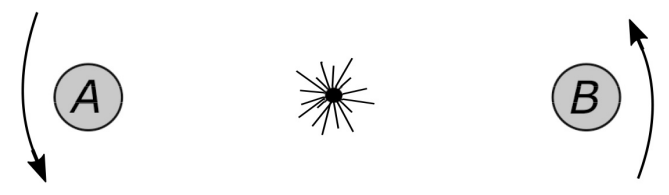

Figure 4. An equal-mass binary star system. A light flashes at their common center of mass. 
From the point of view of $\operatorname{star} A$, star $B$ is moving with speed $v$. Therefore, when the clocks are compared, we should have $t_{B}>t_{A}$. But according to star $B$, star $A$ is moving with speed $v$. Therefore, we should have $t_{A}<t_{B}$, which is another example of the twin paradox, which cannot be resolved by any of the current explanations as the system is completely symmetric.

In addition to these, one can find many other examples in which the process is completely symmetric in spacetime, yet the twin paradox stands and cannot be resolved by the explanations provided in the literature.

\section{Conclusions}

Despite many attempts that have been made to resolve the twin paradox, none of them have been successful. These attempts all resort to a single example, namely the standard version of the twin paradox, in which one twin stays on Earth and the other travels to a distance star and back. One explanation relies on the argument that the traveling twin switches its frame of reference during its motion. But nowhere in the theory of special relativity, it is stated that the reference frame cannot be switched. The second explanation is based on the argument that one twin accelerates where as the other one does not. Again, in the theory of special relativity, there is no discussion that for the time-dilation Equation (1) to be valid the system should not accelerate. In other words, both arguments are based on the unsubstantiated claim of lack of spacetime symmetry in the process.

Furthermore, it has been shown that neither general relativity nor acceleration can explain the twin paradox. The effect still holds as an observer can instantly reverse the direction of motion, maintaining constant speed throughout the entire motion. Alternatively, the moving observer can be thought of as a pair of observers, one traveling away and the other toward the starting point, both with equal constant speeds. They pass each other at the turnaround point, where the clock reading of the first observer is transferred to the second one [19].

In this article, we have shown that the twin paradox stands even when the process is completely symmetric in spacetime, and paradox cannot be resolved by any of the above explanations. Here the authors are not claiming that the twin paradox cannot be solved. All they are trying to do is to bring to the attention of the readers that the explanations provided so far are ad hoc and unacceptable, and that there are many situations where these arguments cannot explain the paradox. If the paradox can ever be resolved, it requires a much deeper understanding of the time dilation concept and the theory of relativity.

\section{Conflicts of Interest}

The authors declare no conflicts of interest regarding the publication of this paper.

\section{References}

[1] Beiser, A. (2003) Concepts of Modern Physics. Sixth Edition, McGraw-Hill, New York, Chapter 1. 
[2] Serway, R.A., Moses, C.J. and Moyer, C.A. (2005) Modern Physics. Third Edition, Brooks/Cole-Thomson Learning, Belmont, Chapter 1.

[3] Ohanian, H.C. and Markert, J.T. (2007) Physics for Engineers and Scientists. Third Edition, Volume 2, Norton and Company, New York, 1224-1229.

[4] Davies, P. (1995) About Time, Einstein's Unfinished Revolution. Simon \& Schuster (Orion Productions), New York, 59-65.

[5] Romer, R.H. (1959) Twin Paradox in Special Relativity. American Journal of Physics, 27, 131. https://doi.org/10.1119/1.1934783

[6] Dray, T. (1990) The Twin Paradox Revisited. American Journal of Physics, 58, 822. https://doi.org/10.1119/1.16373

[7] Debs, T.A. and Redhead, M.L.G. (1996) The Twin "Paradox" and the Conventionality of Simultaneity. American Journal of Physics, 64, 384-392. https://doi.org/10.1119/1.18252

[8] Boughn, S.P. (1989) The Case of the Identically Accelerated Twins. American Journal of Physics, 57, 791-793. https://doi.org/10.1119/1.15894

[9] Halliday, D., Resnick, R. and Walker, J. (2014) Fundamentals of Physics. Tenth Edition, Wiley, New York, Chapter 37.

[10] Einstein, A. (1920) Relativity, the Special and General Theory. Translated by R. W. Lawson, Henry Holt and Company, New York.

[11] Gravitational Time Dilation. https://en.wikipedia.org/wiki/Gravitational time dilation

[12] Hafele, J.C. and Keating, R.E. (1972) Around-the-World Atomic Clocks: Observed Relativistic Time Gains. Science, 177, 168-170. https://doi.org/10.1126/science.177.4044.168

[13] The Twin Paradox Part 1 Stanford University Coursera. https://www.youtube.com/watch?v=1xb-z8ZyvI4

[14] Stuver, A. Einstein's Twin Paradox Explained. https://www.youtube.com/watch?v=h8GqaAp3cGs

[15] How Does Relativity Theory Resolve the Twin Paradox? Scientific American. https://www.scientificamerican.com/article/how-does-relativity-theor

[16] Twin Paradox: The Real Explanation (No Math). https://www.youtube.com/watch?v=noaGNuQCW8A

[17] Twin Paradox: The Real Explanation. https://www.youtube.com/watch?v=GgvajuvSpF4

[18] Complete Solution to the Twins Paradox. https://www.youtube.com/watch?v=0iJZ QGMLD0

[19] Twin Paradox. https://en.wikipedia.org/wiki/Twin_paradox 